Chapter for book edited by C. Pariante and R. M. Nesse. 2009.

Depression: Translational Approaches To Understanding And Treating Oxford University Press (in press)

\title{
Evolutionary genetics of affective disorders
}

\author{
Bernard J. Crespi \\ Department of Biosciences, \\ Simon Fraser University \\ 8888 University Drive, Burnaby BC V5A 1 S6 Canada
}

Correspondence: Bernard Crespi

Department of Biosciences

Simon Fraser University

Burnaby BC V5A 1S6 Canada

email: crespi@sfu.ca

5200 words in main text

Acknowledgements: I am grateful to NSERC and the Canada Council for the Arts for financial support, and to R. Nesse and C. Pariante for motivating and supporting my work on affective disorders. 


\section{Abstract}

I review evolutionary-genetic models for the generation, maintenance and loss of allelic variation underlying polygenic disease, in the context of affective disorders and related conditions. Genetically-based liability to these disorders appears to be due to some combination of mutation-selection balance involving common, small-effect variants and rare, large-effect variants, and natural selection involving antagonistic pleiotropy and balancing selection. At present, the primary usefulness of evolutionary genetics in the study of affective disorders is that it provides important insights into choices of genes, alleles, and haplotypes for analysis via genome-scan and association studies, and motivates a focus on the potential for pleiotropic, beneficial effects of alleles and genotypes that also influence disease risk.

\section{Introduction}

Most evolutionary approaches to the analysis of human cognitive function, affective states, and mental disorders have focused on the potential adaptive significance of the phenotypes involved, in ancestral or modern environments (e. g. Nesse 1999; Nettle 2001, 2004). According to such evolutionary hypotheses, natural selection has optimized human mental phenotypes in the context of functions that are related to reproductive success, and mental disorders have been inferred to represent either unavoidable, maladaptive byproducts of such selection (e. g., Burns 2006), tails of continuous distributions of genetically-based cognitive-affective functional abilities (e. g., Nesse 2004), or manifestations of associations between enhanced abilities in some domain of performance, such as creativity, emotional sensitivity, or propensity to strive for success, and increased risk of mental illness (e. g., Nettle 2001, 2004, 2006a). In this context, major depression and bipolar disorder can be interpreted as forms of dysregulation of adaptive mental systems that regulate mood, where low mood and high mood are normally-adaptive states that contextually-regulate physiology, cognition and behavior to maximize fitness (Nesse 2000; Nettle 2004; Johnson 2005; Wolpert 2008).

Hypotheses for the adaptive significance of human mental traits are extremely difficult to evaluate rigorously, as such tests require quantification of cognitive, affective and behavioral phenotypes in relation to some measures of performance or fitness that are relevant in the context of human evolutionary history. Hypotheses of maladaptation are even more challenging to test, as they require information on genetic, physiological, or developmental 
mechanisms that allows exclusion of hypotheses based on adaptation or adaptive tradeoffs (Crespi 2000; Nesse 2005). Moreover, even when analyses of the functional design and adaptive nature of cognitive and emotional phenotypes yield results consistent with optimization (e. g., Keller and Nesse $2005,2006)$, the implications of such evolutionary, ultimate-level findings for proximate-level analyses of the etiologies of mental disorders, or treatment strategies, often remain unclear (Nesse 1999). As a result, most workers studying or treating affective disorders do so in the absence of evolutionary perspectives, which are viewed as either highly speculative or largely irrelevant.

Adaptive significance is not just a function of phenotypes - it is also a property of the genetic, genomic and epigenetic variation that underlies observed variation in mental traits or other phenotypes. Thus, just as a phenotype may be adaptive or maladaptive in some environmental context, an allele or haplotype may have increased or decreased in frequency as a result of selection over evolutionary time, or it may be maintained at some intermediate frequency. For evolutionary analyses of mental disorders, a focus at the level of alleles and haplotypes offers a number of useful analytic properties, such as the ability to infer the action of population-genetic processes, including selection for advantageous alleles, selection against deleterious alleles, or genetic drift, from data collected in extant populations (e. g., Biswas and Akey 2006; Sabeti et al. 2006; Boyko et al. 2008). Such evolutionary-genetic analyses permit rigorous quantification of how natural selection, drift, mutation, and other processes have influenced genetic liability to affective disorders, which in turn provides direct insights into the proximate genetic, physiological and developmental underpinnings of mental disorders, and the role of optimization by selection in ultimate-level studies of adaptive significance (Crespi et al. 2007).

In this chapter I provide an overview of the evolutionary genetics of affective disorders. My main goal is to integrate results from recent studies of the phenotypic structure and genetic basis of affective and related mental disorders with evolutionary models of the genetic, genomic and epigenetic bases of polygenic human disease. I first define the cognitive-affective phenotypes and psychiatric conditions under consideration, to elucidate their phenotypic architecture, and I review the connections of phenotypic architecture, and formal diagnostic categories, with variation at the genetic level. Second, I attempt to square our knowledge of the genetic basis of affective and other mental disorders with current evolutionary, population-genetic models for the bases of human polygenic disease risk, to assess how well the 'genetics' meets the 'evolution' of mental disorders. Finally, based on the results of these analyses, I suggest how molecular-evolutionary analyses can be applied to 
further our understanding of the etiologies of affective disorders at both the proximate and ultimate levels.

\section{Phenotypic and genetic architecture of psychotic-affective disorders}

Major depression, bipolar disorder, and schizophrenia exhibit broad partial overlap in their constituent behavioral, cognitive, affective, and neurological phenotypes, with gradations between them characterized in terms of schizoaffective disorder, or depression (bipolar or unipolar) with psychotic features (Baethge et al. 2005; Kempf et al. 2005; Boks et al. 2007a; Lin and Mitchell 2008; Smoller et al. 2008). Discussion of the nature of psychological variation between and within these conditions is beyond the scope of this article. However, based on several criteria including patterns of shared phenotypes between disorders, patterns of inheritance within families, and the incidence of these disorders in neurogenetic syndromes (Boks et al. 2007b; Gothelf 2007; Lin and Mitchell 2008), depression, bipolar disorder, and schizophrenia can usefully be characterized as encompassing a 'schizophrenia spectrum', 'psychotic spectrum', or, as described here, 'psychotic-affective spectrum' (Marneros and Akiskal 2006; Crespi and Badcock 2008; Ivleva et al. 2008). These three conditions also partially overlap in their genetic underpinnings (Craddock and Forty 2006; Potash 2006; Blackwood et al. 2007; Farmer et al. 2007; López-León et al. 2007; Owen et al. 2007; Goes et al. 2008), with some genes, such as DISC1 and G72, now known to harbor allelic variants that affect susceptibility to all three disorders (Chubb et al. 2008; Rietschel et al. 2008). Such patterns of partial phenotypic and genetic overlap in psychotic-affective disorders have motivated the hypothesis that major depression, bipolar disorder, and schizophrenia share some genetic risk factors, but are also underlain by genetic variants that are more or less specific to one or two of the disorders (Craddock et al. 2006; Craddock and Forty 2006; Hamshere et al. 2006). The presence of a psychotic-affective spectrum, analogous to the autistic spectrum, indicates that analyses of the evolutionary genetics of affective disorders cannot be easily parsed from analyses of disorders that involve strong elements of psychotic features, mainly schizophrenia.

Conditions on the psychotic-affective spectrum also apparently grade more or less smoothly into normality in non-clinical populations, with schizophrenia grading into schizotypy (Claridge 1997; Fanous et al. 2007; Stefanis et al. 2007), major depression grading into neuroticism (Fullerton et al. 2003; Shifman et al. 2008), and mania in bipolar disorder grading into hypomania and normality (Young, this volume). Such continuous variation in 
phenotypes is indicative of polygenic underpinnings, with many genes, each of small effect, mediating expression (e. g., Rapoport et al. 2005; Tamminga and Holcomb 2005; Craddock et al. 2008; Shifman et al. 2008), and it suggests that genetic liability to psychotic-affective spectrum conditions forms a continuous spectrum with variation in human personality traits (Fanous and Kendler 2004; Nettle 2006a), with important implications for the populationgenetic factors that generate, maintain and remove variation that affects disease risk and phenotypic variation. However, the interface of personality with psychiatric genetics has yet to be sufficiently developed to allow firm inferences to be drawn regarding the nature of associations between normal vs. more-or-less pathological cognitive-affective traits; for example, geneticallymediated personality traits may modulate the clinical presentation of affective disorders, rather than representing direct causal influences on the disorders themselves (Serretti et al. 2006), and depressive personality disorder appears to be at least partially distinct from major depression itself (Ørstavik et al. 2007).

Psychotic-affective spectrum conditions may be caused not just by apparent cumulative and interactive effects of allelic variation with relativelysmall effects, but also by de novo genetic variations of relatively-large effect, either at a genetic scale, such as breakpoints in the DISC1 gene leading to major depression and schizophrenia (Muir et al. 2008), or at a genomic scale, such as gene copy number variation due to duplications or deletions of regions that contain multiple genes. Genomic alterations include, for example, velocardiofacial syndrome due to deletions at 22q11.2, which involves an over20 fold increase in the incidence of schizophrenia, bipolar disorder, and major depression (Gothelf 2007), and Klinefelter syndrome, due to one or more 'extra' $X$ chromosomes in males, which involves a 4-10 fold increased risk of these psychotic-affective spectrum conditions (Boks et al. 2007b). The degree to which such large-scale genomic alterations represent the high end of a continuum of genomic copy-number variants, that grade into smaller, cumulative effects on liability that segregate in populations (rather than arise sporadically, de novo), remains to be determined. However, recent findings that at least $7-10 \%$ of cases of schizophrenia (Walsh et al. 2008; Xu et al. 2008), and autism (Sebat et al. 2007; Marshall et al. 2008), may be associated with copy-number variants suggest that this source of genetic variability contributes substantially to the general incidence of mental disorders.

A third important cause of gene-level variation affecting liability of psychotic-affective conditions, in addition to genetic variants and genomic copy-number variants of large or small effect, is epigenetic variation (Mill and Petronis 2007), especially in genes subject to genomic imprinting (Crespi and 
Badcock 2008). Imprinted genes, which are selectively expressed according to their parent of origin (Tycko and Morison 2002; Haig 2004), comprise only a small proportion of the human genome, but they are disproportionately involved in brain development and function (Davies et al. 2008), and they can be dysregulated in more ways than non-imprinted genes, due to: (1) functional haploidy, such that all allelic variants can have immediate phenotypic effects; (2) alterations in methylation status or histone modifications, in addition to heritable changes in nucleotide sequence affecting expression and function; and (3) the differential involvement of imprinted genes in highly pleiotropic developmental systems mediated in part by antagonistic 'tugs-of-war' between maternally-derived genes and paternally-derived genes (Haig 2004; Smith et al. 2006; Smits and Kelsey 2006; Charalambous et al. 2007; Ubeda and Wilkins 2008). In accordance with these properties of imprinted genes, they exert highly-disproportionate effects on disease risk and progression in, for example, placentation (Fowden et al. 2006) and carcinogenesis (Jelinic and Shaw 2007). The degree to which segregating variation, or de novo alterations, in imprinted genes affect susceptibility to psychotic-affective disorders remains to be determined (Crespi and Badcock 2008; Crespi 2009). However, Prader-Willi syndrome due to uniparental disomy of chromosome 15, which is due to alterations to imprinted gene expression, is the most-penetrant cause of psychotic-affective disorders known to date (Soni et al. 2007, 2008), the imprinted LRRTM1 gene, associated with schizophrenia and schizoaffective disorder, underlies one of the strongest genome-scan linkages to schizophrenia (Francks et al. 2007), and a recent linkage study of anxiety (Middledorp et al. 2008) implicated the imprinted gene cluster at 14q32.2. Crow (2008) has also suggested that epigenetic variation of large effect underlies psychotic-affective disorders, a hypothesis that remains tenable to the degree that genetic and genomic variants continue to explain only a relatively small proportion of inherited or de novo risk.

Genetic, genomic and epigenetic variation exerts its effects on liability to affective-psychotic conditions via alterations to more or less 'normal' neurocognitive architecture. In humans, a primary axis of such architecture is sex, with males and females differing quantitatively in a large suite of neurodevelopmental, neuroanatomical, psychological and other traits (Geary 1998; Baron-Cohen 2003). Sex-specific associations of alleles with some psychotic-affective conditions (e. g., Pickard et al. 2007), and the higher incidence of major depression in females than males (Marcus et al. 2008; Smith et al. 2008), notably implicate sex as an important causal factor in the evolutionary genetics of mental disorders. Crespi and Badcock (2008) suggest that genomic-imprinting effects generate a second axis of human 
cognitive variation, whereby a canalized neurodevelopmental continuum stretches from a bias towards relative effects of maternally-expressed imprinted genes, to dynamically-balanced normality, to a bias towards effects of paternally-expressed imprinted genes. Considered jointly with the axis of male-female variation, variation along such a genomic-imprinting effects axis helps to explain several of the strong observed sex biases in the prevalence and severity of both psychotic-affective and autistic-spectrum conditions (Crespi and Badcock 2008), such as the overall male bias to autism, moresevere autism in females, the overall female bias to depression, and moresevere schizophrenia in males. This model points to genes underlying sex differences in neurodevelopment, and imprinting effects, as important factors in the expression of psychotic-affective conditions, in part via modification of the penetrance of allelic effects (e. g., Zhao et al. 2007); it also suggests that epistatic interactions between imprinted genes and non-imprinted genes involved in sex differentiation, as apparently found in Turner syndrome (Crespi 2008) may play important roles in human development.

\section{Evolutionary-genetic models of psychotic-affective conditions}

The relatively-high heritabilities of neurodevelopmental conditions such as schizophrenia and autism have been considered to pose an evolutionary paradox, given that allelic variants increasing the risk of such conditions should be strongly selected against, resulting in low levels of additive genetic variation maintained in populations (Keller and Miller 2006). Three main categories of evolutionary-genetic model have been proposed thus far to explain such an apparent paradox in the maintenance of alleles for polygenic disease risk (Table 1):

(1) models based on a large number of slightly-deleterious variants, most of small effect, subject to inputs via mutation across many loci, maintenance by drift under sufficiently weak selection or small population size, and removal via purifying selection (Hughes et al. 2003; Kryukov et al. 2007; Bodmer and Bonilla 2008);

(2) models based on the effects of rare, more highly-deleterious genetic or genomic variants, with common variants of small effect exerting a relativelysmall influence on overall disease risk (Fearnhead et al. 2004; Zhao et al. 2007; Bodmer and Bonilla 2008; Walsh et al. 2008); and 
(3) models based on evolutionary advantages, now or in the past, of alleles that also increase disease risk (Kryukov et al. 2007).

The first model corresponds to the common disease-common variants hypothesis (Lohmueller et al. 2003), whereby polygenic disease is due to cumulative and interactive combinations of many deleterious alleles, each of small effect, in the context of the myriad mutational targets provided by large numbers of genes, large mutation-prone genes, and complex pathways (e. g., Smith et al. 2006); by contrast, under the second model, segregating genetic variation in disease risk has minor effects compared to larger-effect, de novo variants. Variation is maintained under the third model by any of a number of selective processes, such as balancing selection or antagonistic pleiotropy, that can maintain risk alleles at non-trivial frequencies (Keller and Miller 2006; Kryukov et al. 2007). These models are not mutually exclusive, and the first two models may grade into one another given continuous distributions of frequencies for disease risk alleles across loci, effect sizes, and levels of penetrance (Boyko et al. 2008). Moreover, effects of genetic drift may interact with effects of purifying selection, by generating increases in the frequencies of more-deleterious variants in relatively-small groups, such as human founder populations (e. g., Fearnhead et al. 2004; Lohmueller et al. 2008; Boyko et al. 2008). How well do these models with the available data, and what are the implications for elucidating the genetic bases of neurodevelopmental disorders?

\section{(a) Common or rare alleles subject to purifying selection}

Slightly-deleterious alleles can be maintained at appreciable frequencies, across many loci, when mutations to such alleles are common across the genome and purifying selection is relatively weak. If risk alleles exhibit appreciable allele frequencies and small effect sizes, then the common variant common disease paradigm described for schizophrenia, bipolar disorder, and other diseases by Lohmueller et al. (2003) holds, but if disease is mediated predominantly by rare alleles, then current molecular-psychiatric strategies for identifying disease loci, such as whole-genome scans that probe relativelycommon variants, will be largely ineffective (Hughes 2007; Bodmer and Bonilla 2008; Boyko et al. 2008). Kryukov et al. (2007) integrated information from known human disease mutations, human-chimp divergences, and human genetic variation to show that up to $70 \%$ of low-frequency missense mutations are deleterious, which implies that mutation-selection balance of low-frequency alleles is a feasible population genetic mechanism for the maintenance of common human polygenic diseases (see also Fay et al. 2001). For psychotic- 
affective disorders, these inferences are consistent with results from five recent studies: (1) a higher frequency of rare variants, for six schizophrenia-associated genes, in patients than in controls (Winantea et al. 2006); (2) the presence of rare variants of the SYNGR1 gene in schizophrenia patients but not controls (Cheng and Chen 2007); (3) the observation that over $60 \%$ of cases of schizophrenia in Daghestan genetic isolates were offspring of consanguineous marriages (Bulayeva et al. 2005); (4) a significantly-higher incidence of rare, large-haplotype, recessive genotypes in schizophrenia patients than controls (Lencz et al. 2007); and (5) the presence of rare, high-risk allelic variants detected in a study of the role of DISC1 alleles in schizophrenia risk (Song et al. 2008). These convergent findings should motivate genotyping strategies that detect a higher proportion of low-frequency variants, given their potential cumulative role in disease risk, and the resultant data sets will also allow robust estimation of Tajima's D-statistic (e. g., Winantea et al. 2006), whereby evidence for purifying selection, balancing selection, and positive selection can be inferred from the distribution of allele-frequency variation (Bamshad and Wooding 2003; Biswas and Akey 2006).

Regardless of the spectrum of allele frequencies for risk alleles, there is now considerable population-genetic evidence for the presence of abundant, slightly-deleterious variants in humans, many of which are apparently subject to purifying selection (Bustamante et al. 2005; Hughes et al. 2003, Yampolsky et al. 2005; Hughes 2007; Lohmueller et al. 2008). Such purifying selection has been detected, for example, via quantification of global reductions in human among-population differentiation for amino-acid altering mutational sites, especially for disease-related genes (Barreiro et al. 2008). Perhaps the most important implication of these findings is that identification of sites that are likely subject to purifying selection provides a strong clue to functional significance, allowing efficient, evolutionarily-informed choices of SNPs and other variants for linkage or association studies (Hughes 2007). Such studies will in turn determine the degree to which mutation-selection balance across many loci can cumulatively account for a substantial proportion of disease risk, for common diseases in general and psychotic-affective disorders in particular.

\section{(b) Rare genomic alterations subject to strong purifying selection}

An important role for individually-rare, highly-deleterious genomic variants is provided by Walsh et al. (2008) and Xu et al. (2008), who reported notably higher frequencies of rare, high-penetrance structural genomic changes (duplications and deletions) in schizophrenia cases than in controls (e. g., 15$20 \%$ vs $5 \%$ in Walsh et al. 2008). These findings concord with recent studies of 
autism reporting similarly increased frequencies of copy-number variants in patients (Sebat et al. 2007; Marshall et al. 2008), with a stronger advanced paternal-age effect in sporadic than familial schizophrenia (Sipos et al. 2004), and with a recent genetic model of autism that posits a high incidence of de novo large-effect mutations with higher penetrance in males than females (Zhao et al. 2007). By this model, most of the genes thus far implicated in neurodevelopmental disorders via association or linkage studies have been suggested to represent modifier loci of small effect that are largely irrelevant to the risk of disease (Zhao et al. 2007; Walsh et al. 2008). An important corollary of this model is that only alleles of relatively large effect and penetrance will result in familial concentration of disease cases (Bodmer and Bonilla 2008), a pattern commonly found in psychotic-affective disorders (e. g., Potash et al. 2001; Schurhoff et al. 2003); this pattern may be interpreted as broadly concordant with the rare, large-effect deleterious-allele model for genes or copynumber variants, or as evidence that other population-genetic factors are involved.

These recent studies are of considerable importance in revealing that gene copy-number variation mediates a considerable proportion of the risk for neurodevelopmental disorders (Cantor and Geschwind 2008), although bipolar disorder and major depression have yet to be studied in this context. Such analyses have, however, focused on relatively-severe cases (such as childhood-onset schizophrenia, and Kanner autism), such that the expected role of copy-number variants in less-severe conditions, such as high-functioning autism, bipolar II, or mild unipolar depression, remains unclear. More generally, the effects of relatively-common copy-number variants in modulating dimensional variation in neurodevelopmental conditions and cognitive phenotypes have yet to be assessed. Evidence for functional, adaptive effects of such variants comes from Sharp et al. (2005), who show that genomic copy number polymorphisms are common in humans and likely mediate normal variation as well as disease, and from Hahn et al. (2007) and Jiang et al. (2007), who describe evidence for an accelerated rate of evolution in gene copy number among primates and along the human lineage, driven in part by positive selection on brain-related genes.

\section{(c) Common alleles subject to positive selection or balancing selection}

Alleles or genotypes that increase susceptibility to affective disorders may be maintained in human populations because they are, or have been, advantageous in some context other than disease. The primary models based on effects of positive selection (selection 'for' particular genetic variants, that 
arise and sweep through populations to some frequency; Sabeti et al. 2006; Hughes 2007) that have been proposed include: (1) changing selection pressures, such that common alleles that were advantageous in ancestral environments (for example, 'thrifty' genes affecting the regulation of metabolism) are now deleterious, and derived alleles are selected for (Di Rienzo and Hudson 2005; Di Rienzo 2006), (2) balancing selection, whereby individuals with heterozygous genotypes are favored, resulting in the maintenance at high frequency of deleterious homozygotes, and (3) antagonistic pleiotropy, whereby selected alleles exert positive effects in one context, or early in the lifespan, that are stronger than, or balanced by, negative effects expressed in some other context or later in life (Keller and Miller 2006; Kryukov et al. 2007).

The ancestral-susceptibility model has been supported by data from molecular-evolutionary, geographic and physiological analyses of genes involved in risk of hypertension, type 2 diabetes, and several other common human diseases (e. g., Di Rienzo and Hudson 2005; Young et al. 2005; Helgason et al. 2007). Lo et al. (2007) describe evidence that this model may apply in the context of schizophrenia-protective haplotypes of the GABRB2 gene being subject to recent positive selection, although in this case the causes and context of selection remain to be elucidated. More generally, an ancestralsusceptibility model for mental disorders must posit and evaluate roles for specific environmental or epistatic effects that mediate large-scale shifts in how specific alleles influence disease risk. The most obvious difference between ancestral and modern human environments, as regards susceptibility to affective disorders, is the social environment shifting away from villages and extended families towards the novel, large social groups of cities and the novel, small groupings of more or less isolated nuclear families. However, reductions in the strength of social support networks might be expected to simply increase depression risk in modern environments, rather than change the direction or nature of gene by environment interactions. Alternatively, the human social environment may have qualitatively changed, in ways related to increased levels of stress and complexity.

Evidence for balancing selection among genotypes, for genes whose variants influence susceptibility of psychotic-affective disorders, is limited to studies that show effects of heterosis for such genes as DRD2, DRD3, DRD4, HTR2A, SLC6A4 and TPH1, in some physiological and behavioral contexts (e. g., Comings and MacMurray 2000; Reuter and Hennig 2005). The general paucity of population-genetic evidence for balancing selection on genes underling mental disorders may arise in part from ascertainment bias in SNPbased scans for selection, such that high-frequency variants are differentially detected (Kelley and Swanson 2008). At the phenotypic and epidemiological 
levels, psychotic-affective cognition or disorders have been associated with measures of enhanced creativity (Nettle 2001; Burch et al. 2006), mating success (Nettle and Clegg 2006), socio-economic achievement (Jamison 1996; Johnson 2005) and, in several studies, higher fertility of first-order relatives (reviewed in Crespi et al. 2007; Crespi and Badcock 2008), compared to controls. These findings are consistent with evolutionary benefits of alleles and genotypes underlying such disorders but they do not directly or quantitatively address any particular model based on positive or balancing selection.

Clear evidence for antagonistic pleiotropy of alleles underlying psychoticaffective disorders has been reported for APOE genotypes, such that the E4 allele, linked with higher risk of major depression (Yen et al. 2007) and bipolar disorder (Bellivier et al. 1997) is also associated with enhanced verbal skills in childhood and other potential early-life benefits (Alexander et al. 2007). Similarly, the schizophrenia-risk haplotype of DAOA, a gene additionally linked with bipolar disorder (Prata et al. 2007), is also associated with enhanced semantic fluency (Opgen-Rhein et al. 2008), and the schizophrenia-risk haplotype of the PPP1R1B is associated with measures of enhanced frontostriatal function (Meyer-Lindenberg et al. 2007). The generality of a role for antagonistic pleiotropy in psychotic-affective conditions remains unclear, primarily due to lack of study of this mechanism outside the context of senescence theory (e. g., Capri et al. 2006). Pleiotropy itself is a virtually universal mode of gene action (e. g., Knight et al. 2006; Barriero et al. 2008), and most of the genes involved in neurodevelopment and neurological function are known to be fundamentally involved in other processes as well (Kendler 2005), such as mediation of tumor suppression by the APC and TP53 genes, both of which have been implicated in risk of psychotic-affective disorders (Cui et al. 2005; Ni et al. 2005) and have also apparently been subject to positive selection in the human lineage (Voight et al. 2006; Crespi et al. 2007). Another example of pleiotropic effects in 'neuronal' genes is testicular functions in males, given strong patterns of gene co-expression in brain and testis (Guo et al. 2005) that are apparently underlain by shared receptor functions such as exocytosis (Meizel 2004). Genes differentially expressed in testis exhibit notably-enhanced signatures of positive selection in humans (Nielsen et al. 2005), selection that may generate negative pleiotropic effects in the context of neuronal activities (Qin et al. 2007).

The primary evidence congruent with a hypothesis of positive selection having some role in the evolutionary-genetic basis of psychotic affective disorders, either in increasing, maintaining or reducing levels of genetic variation, is that a considerable number of the genes that have been linked with schizophrenia, bipolar disorder, major depression, and other diseases (Barriero 
et al. 2008), show evidence of positive selection in recent human evolution. These include some of the best-supported candidate genes for affective disorders (Table 2), and for schizophrenia (Crespi et al. 2007), such as DTNBP1, NRG1, and SLC6A4, and schizophrenia-associated genes show a statistically-stronger signal of positive selection than a control set of genes involved in neuronal activities (Crespi et al. 2007). A variety of hypotheses may potentially explain the phenotypic basis of this molecular-evolutionary pattern, including: (1) positive selection for alleles protective against mental disorders or reduced cognitive-affective function (e. g., Weiss et al. 2007); (2) antagonistic pleiotropy, such that advantageous effects of selected alleles or genotypes in some context, such as creativity or social-emotional cognition, are not outweighed by an increased risk of mental disorders conferred by these same alleles, across evolutionary time (Nettle 2001; Burns 2006; Crespi et al. 2007), or (3) positive selection in contexts unrelated to mental disorders, given the highly-pleiotropic nature of most of the genes involved. These hypotheses can be evaluated in two main ways:

(1) Genes, alleles, and haplotypes inferred as being subject to positive selection in humans can be chosen as markers to evaluate their potential associations with the risk of psychotic-affective or other polygenic disorders (Thomas and Kejariwal 2004; Crespi et al. 2007). The beauty of this approach is that alleles subject to positive selection are predicted to be functional (McVean and Spencer 2006), which should increase the likelihood that associations will be ascertained (Biswas and Akey 2006). Such studies can also be conducted in the framework of which haplotypes are ancestral vs. derived in humans ancestry, to infer the validity of the ancestral-susceptibility model in the context of mental diseases (e. g., Weiss et al. 2007);

(2) Positively-selected SNPs and haplotypes, and disease-associated alleles, can each be tested in relation to multiple cognitive-affective phenotypes (e. g., Comings et al. 2003; Meyer-Lindenberg et al. 2007) including those that may be beneficial, rather than focusing exclusively on deficits and potential disease endophenotypes. For example, two recent studies have indeed shown that schizophrenia-risk alleles (for the SLC6A4 and TPH1 genes) were pleiotropically-associated with measures of increased creativity (BachnerMelman et al. 2005; Reuter et al. 2006). Such studies allow testing of the idea that having some moderate number of alleles associated with risk of psychoticaffective disorders, across many loci, leads to enhancements in some cognitive domains, while having 'too many' such alleles increases risk of disease (Nesse 2004). 
A key consideration regarding the evaluation of alternative yet nonexclusive models for the evolutionary-genetic forces affecting human diseaserelated genes is that formal models for the maintenance of variation generally assume equilibria between opposing population-genetic processes (Di Rienzo 2006), yet there is no good reason to expect that modern human populations are in equilibrium (Lohmueller et al. 2008). Indeed, Hawks et al. (2007) describe population-genomic evidence that humans are currently undergoing an acceleration of adaptive evolution, due to a combination of larger population sizes (creating more potential targets for adaptive mutation), expanding populations (which increase the fixation probabilities of adaptive mutations), and more-rapid environmental change (which generates stronger selection itself). To the extent that such environmental change involves aspects of human social and emotional cognition that mediate reproductive success, positive selection on genes affecting liability to mental disorders is an expected consequence. It is also of critical importance to avoid a false dichotomy between models based on positive or balancing selection, and models invoking mutation-selection balance (Nettle 2006b), because the processes are not incompatible and studies conducted to date provide clear evidence for both (Fay et al. 2001; Bustamante et al. 2005; Keller and Miller 2006; Crespi et al. 2007; Barriero et al. 2008).

\section{Conclusions}

The fields of molecular-genetic psychiatry and human evolutionary genetics have burgeoned over the past 10 years but continue to develop in virtual isolation. Study of the evolutionary genetics of psychotic-affective disorders is thus in its infancy, but it holds enormous promise for future progress in both domains. At present, the primary usefulness of evolutionary-genetic tools for elucidation of the etiologies of such disorders is that they provide windows into the expected functionality of specific genetic variants as either positivelyselected, subject to balancing selection, or subject to weak purifying selection, they provide a temporal dimension to the distribution of human disease-related genetic variation, and they provide insights into which methods are most likely to efficiently detect loci and alleles underlying disease. As firm evidence accumulates regarding the set of genes and alleles mediating the expression of mental disorders, functional information in particular becomes increasingly important in forging links from genetic, genomic and epigenetic variation to physiology, neurodevelopment, cognition, and behavior (Kelley and Swanson 2008). Evidence regarding the adaptive significance of genetic variants underlying susceptibility to psychotic-affective disorders will also allow much 
more robust evaluation of hypotheses that posit selective benefits to phenotypes associated, in less extreme form, with disorders of cognition and affect. 
Table 1. Three non-exclusive evolutionary-genetic models may help to explain the role of different population-genetic forces in the origin, maintenance, and loss of genetic variation that underlies susceptibility to affective disorders, and each of these models has important implications for future analyses.

Evolutionary-genetic model

Many variants, each of small effect; variants rare or common
Population-genetic forces
Implications for future studies
Weak purifying selection on de novo and segregating variants

Strong purifying selection, mainly on de novo variants
Rare variants, each of large effect

Common variants, each of small to moderate effect
Positive selection, antagonistic pleiotropy, balancing selection
Target alleles apparently subject to weak purifying selection; analyze common and rare variants in association and genome scan studies

Most known risk variants of small effect are more or less irrelevant; systematically target copy number variants and rare alleles

Target alleles and haplotypes apparently subject to selection test for beneficial effects of risk and protective variants 
Table 2. Evidence of positive selection is available for each of a large suite of genes that harbor variants implicated in bipolar disorder (most genes), major depression, and schizoaffective disorder. Details of the Voight et al. (2006) results are available in the Haplotter web interface, and Crespi et al. (2007, Table 4) collates relevant information on positive selection from previous studies. Most of the genetic-association citations are available from Carter (2007) and Crespi et al. (2007); additional citations are available upon request. Genes in boldface exhibit especially well-replicated associations, and many of the genes have also been associated with schizophrenia risk. Alleles or haplotypes that have been inferred as subject to positive selection in humans are especially likely to exhibit functional effects on affect and cognition, and so may be especially useful targets for molecular-psychiatric analyses.

Gene

Evidence for positive selection in humans
ALG9
Voight et al. (2006)
APOE
Crespi et al. (2007, Table 4)
BRD1
Voight et al. (2006)
CABIN1
Voight et al. (2006)
CAPON
Voight et al. (2006)
CLOCK
Voight et al. (2006)
DISC1
DOCK 9
Crespi et al. (2007)(human-chimp lineage)
DRD4
Voight et al. $(2006)(p=0.0504)$
DTNBP1
GABRA1
Crespi et al. (2007, Table 4)
GRM3
HMG2L1
IMPA2
Voight et al. (2006), Crespi et al. (2007, Table 4)
Voight et al. (2006)
LRRTM1
Voight et al. (2006), Crespi et al. (2007, Table 4)
Voight et al. (2006)
MAOA
Voight et al. (2006)
NPAS3
NR3C1
Voight et al. (2006), Francks et al. (2007)
Crespi et al. (2007, Table 4)
NR3C2
NRG1
Crespi et al. (2007, Table 4)
PAX1
Bustamante et al. (2005)
Bustamante et al. (2005) $(p=0.068)$
Crespi et al. (2007)
Bustamante et al. (2005) 
Table 2 (continued)

Gene Evidence for positive selection in humans

$\begin{array}{ll}\text { PDE11A } & \text { Voight et al. (2006) } \\ \text { PDLIM5 } & \begin{array}{l}\text { Crespi et al. 2007, Table 4), Voight et al. (2006) } \\ \text { (selected SNPs, not gene-wide significant) }\end{array} \\ \text { PIK3C3 } & \text { Voight et al. (2006) } \\ \text { PLCG1 } & \text { Voight et al. (2006) } \\ \text { RFX4 } & \text { Voight et al. (2006) } \\ \text { SLC12A6 } & \text { Voight et al. (2006) } \\ \text { SLC6A3 } & \text { Bustamante et al. (2005)(p=0.06) } \\ \text { SLC6A4 } & \text { Bustamante et al. (2005), Voight et al. (2006) } \\ \text { SYNGR1 } & \text { Voight et al. (2006) } \\ \text { SYNJ1 } & \text { Voight et al. (2006) } \\ \text { TOM1 } & \text { Voight et al. (2006) } \\ \text { TPH1 } & \text { Bustamante et al. }(2005)(p=0.06)\end{array}$




\section{References}

Alexander, D.M., Williams, L.M., Gatt, J.M. et al. (2007). The contribution of apolipoprotein $E$ alleles on cognitive performance and dynamic neural activity over six decades. Biological Psychology, 75, 229-38

Bachner-Melman, R., Dina, C., Zohar, A.H. et al. (2005). AVPR1a and SLC6A4 gene polymorphisms are associated with creative dance performance. PLoS Genetics, 1, e42

Baethge, C., Baldessarini, R.J., Freudenthal, K., Streeruwitz, A., Bauer, M. and Bschor, T. (2005). Hallucinations in bipolar disorder: characteristics and comparison to unipolar depression and schizophrenia. Bipolar Disorder, 7, $136-45$

Bamshad, M. and Wooding, S.P. (2003). Signatures of natural selection in the human genome. Nature Reviews Genetics, 4, 99-111

Baron-Cohen, S. (2003). The Essential Difference: The Truth about The Male and Female Brain. New York: Basic Books/Penguin

Barreiro, L.B., Laval, G., Quach, H., Patin, E. and Quintana-Murci, L. (2008). Natural selection has driven population differentiation in modern humans. Nature Genetics, 40, 340-5

Bellivier, F., Laplanche, J.L., Schürhoff, F., Feingold, J., Féline, A., Jouvent, R., Launay, J.M., and Leboyer, M. (1997). Apolipoprotein E gene polymorphism in early and late onset bipolar patients. Neuroscience Letters, 233, 45-8

Biswas, S. and Akey, J.M. (2006). Genomic insights into positive selection. Trends in Genetics, 22, 437-46

Blackwood, D.H., Pickard, B.J., Thomson, P.A., Evans, K.L., Porteous, D.J. and Muir, W.J. (2007). Are some genetic risk factors common to schizophrenia, bipolar disorder and depression? Evidence from DISC1, GRIK4 and NRG1. Neurotoxicity Research, 11, 73-83

Bodmer, W. and Bonilla, C. (2008). Common and rare variants in multifactorial susceptibility to common diseases. Nature Genetics, 40, Number 6, 695-701

Boks, M.P., Leask, S., Vermunt, J.K. and Kahn, R.S. (2007a). The structure of psychosis revisited: the role of mood symptoms. Schizophrenia Research, 93, 178-85

Boks, M.P., de Vette, M.H., Sommer, I.E. et al. (2007b). Psychiatric morbidity and X-chromosomal origin in a Klinefelter sample. Schizophrenia Research, 93, 399-402 
Burch, G.S., Pavelis, C., Hemsley, D.R. and Corr, P.J. (2006). Schizotypy and creativity in visual artists. British Journal of Psychology, 97, 177-90

Burns, J.K. (2006). Psychosis: a costly by-product of social brain evolution in Homo sapiens. Progress in Neuro-Psychopharmacology and Biological Psychiatry, 30, 797-814

Bustamante, C.D., Fledel-Alon, A., Williamson, S. et al. (2005). Natural selection on protein-coding genes in the human genome. Nature, 437, 11537

Cantor, R.M. and Geschwind, D.H. (2008). Schizophrenia: genome, interrupted. Neuron, 58, 165-7

Capri, M., Salvioli, S., Sevini, F. et al. (2006). The genetics of human longevity. Annals of the New York Academy of Sciences, 1067, 252-63

Carter, C.J. (2007). Multiple genes and factors associated with bipolar disorder converge on growth factor and stress activated kinase pathways controlling translation initiation: implications for oligodendrocyte viability. Neurochemistry International, 50, 461-90

Charalambous, M., da Rocha, S.T. and Ferguson-Smith, A.C. (2007). Genomic imprinting, growth control and the allocation of nutritional resources: consequences for postnatal life. Current Opinions in Endocrinology, Diabetes and Obesity, 14, 3-12

Cheng, M.C. and Chen, C.H. (2007). Identification of rare mutations of synaptogyrin 1 gene in patients with schizophrenia. Journal of Psychiatric Research, 41, 1027-31

Chubb, J.E., Bradshaw, N.J., Soares, D.C., Porteous, D.J. and Millar, J.K. (2008). The DISC locus in psychiatric illness. Molecular Psychiatry, 13, 3664

Claridge, G., editor. (1997). Schizotypy: Implications for IIIness and Health. Oxford, UK: Oxford University Press

Comings, D.E., Gonzalez, N.S., Cheng Li, S.C. and MacMurray, J. (2003). A "line item" approach to the identification of genes involved in polygenic behavioral disorders: the adrenergic alpha2A (ADRA2A) gene. American Journal of Medical Genetics. Part B, Neuropsychiatric Genetics, 118, 110-4

Comings, D.E. and MacMurray, J.P. (2000). Molecular heterosis: a review. Molecular Genetics and Metabolism, 71, 19-31

Craddock, N. and Forty, L. (2006). Genetics of affective (mood) disorders. European Journal of Human Genetics. 14, 660-8

Craddock, N., O'Donovan, M.C. Owen, M.J. (2006). Genes for schizophrenia and bipolar disorder? Implications for psychiatric nosology. Schizophrenia Bulletin, 32, 9-16 
Craddock, N., O'Donovan, M.C. and Owen, M.J. (2008). Genome-wide association studies in psychiatry: lessons from early studies of nonpsychiatric and psychiatric phenotypes. Molecular Psychiatry, (in press).

Crespi, B. (2000). The evolution of maladaptation. Heredity, 84, 623-9

Crespi, B. (2008). Turner syndrome and the evolution of human sexual dimorphism. Evolutionary Applications, (in press)

Crespi, B. (2009). Genomic imprinting in the development and evolution of psychosis. Biological Reviews, (in press)

Crespi, B. and Badcock, C. (2008) Psychosis and autism as diametrical disorders of the social brain. Behavioral and Brain Sciences. (in press)

Crespi, B., Summers, K. and Dorus, S. (2007). Adaptive evolution of genes underlying schizophrenia. Proceedings of the Royal Society of London Series B, Biological Sciences, 274, 2801-10

Crow, T.J. (2008). Craddock \& Owen vs Kraepelin: 85 years late, mesmerised by "polygenes." Schizophrenia Research, (in press)

Cui, D.H., Jiang, K.D., Jiang, S.D., Xu, Y.F. and Yao, H. (2005). The tumor suppressor adenomatous polyposis coli gene is associated with susceptibility to schizophrenia. Molecular Psychiatry, 10, 669-77

Davies, W., Isles, A. R., Humby, T. and Wilkinson, L. S. (2008). What are imprinted genes doing in the brain? Epigenetics, 2, 201-6

Di Rienzo, A. (2006). Population genetics models of common diseases. Current Opinion in Genetics \& Development, 16, 630-6

Di Rienzo, A. and Hudson, R.R. (2005). An evolutionary framework for common diseases: the ancestral-susceptibility model. Trends in Genetics, 21, 596-601

Fanous, A.H. and Kendler, K.S. (2004). The genetic relationship of personality to major depression and schizophrenia. Neurotoxicity Research, 6, 43-50

Fanous, A.H., Neale, M.C., Gardner, C.O. et al. (2007). Significant correlation in linkage signals from genome-wide scans of schizophrenia and schizotypy. Molecular Psychiatry, 12, 958-65

Farmer, A., Elkin, A. and McGuffin, P. (2007). The genetics of bipolar affective disorder. Current Opinion in Psychiatry, 20, 8-12

Fay, J.C., Wyckoff, G.J. and Wu, C.I. (2001). Positive and negative selection on the human genome. Genetics, 158, 1227-34

Fowden, A.L., Sibley, C., Reik, W. and Constancia, M. (2006). Imprinted genes, placental development and fetal growth. Hormone Research, 65 Suppl 3, 50-8

Francks, C., Maegawa, S., Laurén, J. et al. (2007). LRRTM1 on chromosome $2 \mathrm{p} 12$ is a maternally suppressed gene that is associated paternally with handedness and schizophrenia. Molecular Psychiatry, 12, 1129-39 
Fullerton, J., Cubin, M., Tiwari, H. et al. (2003). Linkage analysis of extremely discordant and concordant sibling pairs identifies quantitative-trait loci that influence variation in the human personality trait neuroticism. American Journal of Human Genetics, 72, 879-90

Geary, D.C. (1998). Male, Female: The Evolution of Human Sex Differences. Washington, DC: American Psychological Association

Goes, F.S., Sanders, L.L. and Potash, J.B. (2008). The genetics of psychotic bipolar disorder. Current Psychiatry Reports, 10, 178-89

Gothelf, D. (2007). Velocardiofacial syndrome. Child and Adolescent Psychiatric Clinics of North America, 16, 677-93

Guo, J.H., Huang, Q., Studholme, D.J., Wu, C.Q. and Zhao, Z. (2005).

Transcriptomic analyses support the similarity of gene expression between brain and testis in human as well as mouse. Cytogenetic and Genome Research, 111, 107-9

Hahn, M.W., Demuth, J.P. and Han, S.G. (2007). Accelerated rate of gene gain and loss in primates. Genetics, 177, 1941-9

Haig, D. (2004). Genomic imprinting and kinship: how good is the evidence? Annual Reviews of Genetics, 38, 553-85

Hamshere, M.L., Williams, N.M., Norton, N. et al. (2006). Genome wide significant linkage in schizophrenia conditioning on occurrence of depressive episodes. Journal of Medical Genetics, 43, 563-7

Hawks, J., Wang, E.T., Cochran, G.M., Harpending, H.C. and Moyzis, R.K. (2007). Recent acceleration of human adaptive evolution. Proceedings of the National Academy of Sciences USA, 104, 20753-8

Helgason, A., Pálsson, S., Thorleifsson, G. et al. (2007). Refining the impact of TCF7L2 gene variants on type 2 diabetes and adaptive evolution. Nature Genetics, 39, 218-25

Hughes, A.L. (2007). Looking for Darwin in all the wrong places: the misguided quest for positive selection at the nucleotide sequence level. Heredity, 99, 364-73

Hughes, A.L., Packer, B., Welch, R., Bergen, A.W., Chanock, S.J. and Yeager, M. (2003). Widespread purifying selection at polymorphic sites in human protein-coding loci. Proceedings of the National Academy of Sciences USA, 100, 15754-7

Ivleva, E., Thaker, G. and Tamminga C.A. (2008). Comparing genes and phenomenology in the major psychoses: schizophrenia and bipolar 1 disorder. Schizophrenia Bulletin, (in press)

Jamison, K.R. (1996). Touched with Fire: Manic-Depressive Illness and the Artistic Temperament. New York: The Free Press 
Jelinic, P. and Shaw, P. (2007). Loss of imprinting and cancer. Journal of Pathology, 211, 261-8

Jiang, Z., Tang, H., Ventura, M. et al. (2007). Ancestral reconstruction of segmental duplications reveals punctuated cores of human genome evolution. Nature Genetics, 39, 1361-8

Johnson, S.L. (2005). Mania and dysregulation in goal pursuit: a review. Clinical Psychology Review, 25, 241-62

Kelley, J.L. and Swanson, W.J. (2008). Positive selection in the human genome: from genome scans to biological significance. Annual Review of Genomics and Human Genetics, (in press).

Keller, M.C. and Miller, G. (2006). Resolving the paradox of common, harmful, heritable mental disorders: which evolutionary genetic models work best? Behavioral and Brain Sciences, 29, 385-404

Keller, M.C. and Nesse, R.M. (2005). Is low mood an adaptation? Evidence for subtypes with symptoms that match precipitants. Journal of Affective Disorders, 86, 27-35

Keller, M.C. and Nesse, R.M. (2006). The evolutionary significance of depressive symptoms: different adverse situations lead to different depressive symptom patterns. Journal of Personality and Social Psychology, 91, 316-30

Kempf, L., Hussain, N. and Potash, J.B. (2005). Mood disorder with psychotic features, schizoaffective disorder, and schizophrenia with mood features: trouble at the borders. International Review of Psychiatry, 17, 9-19

Kendler, K.S. (2005). "A gene for...": the nature of gene action in psychiatric disorders. The American Journal of Psychiatry, 162, 1243-52

Knight, C.G., Zitzmann, N., Prabhakar, S., et al. (2006). Unraveling adaptive evolution: how a single point mutation affects the protein coregulation network. Nature Genetics, 38, 1015-22

Kryukov, G.V., Pennacchio, L.A. and Sunyaev, S.R. (2007). Most rare missense alleles are deleterious in humans: implications for complex disease and association studies. American Journal of Human Genetics, 80, 727-39

Lencz, T., Lambert, C., DeRosse, P., Burdick, K.E., Morgan, T.V., Kane, J.M., Kucherlapati, R., and Malhotra, A.K. (2007). Runs of homozygosity reveal highly penetrant recessive loci in schizophrenia. Proceedings of the National Academy of Sciences U. S. A., 104, 19942-7

Lin, P.I. and Mitchell, B.D. (2008). Approaches for unraveling the joint genetic determinants of schizophrenia and bipolar disorder. Schizophrenia Bulletin, (in press) 
Lo, W.S., Xu, Z., Yu, Z. et al. (2007). Positive selection within the schizophrenia-associated $\mathrm{GABA}(\mathrm{A})$ receptor beta2 gene. PLoS ONE, 2, e462

Lohmueller, K.E., Pearce, C.L., Pike, M., Lander, E.S. and Hirschhorn, J.N. (2003). Meta-analysis of genetic association studies supports a contribution of common variants to susceptibility to common disease. Nature Genetics, 33, 177-82

Lohmueller, K.E., Indap, A.R, Schmidt, S., et al. (2008). Proportionally more deleterious genetic variation in European than in African populations. Nature, 451, 994-7

López-León, S., Janssens, A.C., González-Zuloeta Ladd, A.M. et al. (2007). Meta-analyses of genetic studies on major depressive disorder. Molecular Psychiatry, (in press)

Marcus, S.M., Kerber, K.B., Rush, A.J. et al. (2008). Sex differences in depression symptoms in treatment-seeking adults: confirmatory analyses from the Sequenced Treatment Alternatives to Relieve Depression study. Comprehensive Psychiatry, 49, 238-46

Marneros, A. and Akiskal, H.S. eds. (2006). The Overlap of Affective and Schizophrenic Spectra. New York: Cambridge University Press

Marshall, C.R., Noor, A., Vincent, J.B. et al. (2008). Structural variation of chromosomes in autism spectrum disorder. American Journal of Human Genetics, 82, 477-88

McVean, G. and Spencer, C.C. (2006). Scanning the human genome for signals of selection. Current Opinion in Genetics \& Development, 16, 624-9

Meizel, S. (2004). The sperm, a neuron with a tail: 'neuronal' receptors in mammalian sperm. Biological Reviews Cambridge Philosophical Society, 79, 713-32

Meyer-Lindenberg, A., Straub, R.E., Lipska, B.K. et al. (2007). Genetic evidence implicating DARPP-32 in human frontostriatal structure, function, and cognition. Journal of Clinical Investigation, 117, 672-82

Middeldorp, C.M., Hottenga, J.J., Slagboom, P.E. et al. (2008). Linkage on chromosome 14 in a genome-wide linkage study of a broad anxiety phenotype. Molecular Psychiatry, 13, 84-9

Mill, J. and Petronis, A. (2007). Molecular studies of major depressive disorder: the epigenetic perspective. Molecular Psychiatry, 12, 799-814

Muir, W.J., Pickard, B.S. and Blackwood, D.H. (2008). Disrupted-inSchizophrenia-1. Current Psychiatry Reports, 10, 140-7

Nesse, R.M. (1999). Proximate and evolutionary studies of anxiety, stress and depression: synergy at the interface. Neuroscience \& Biobehavioral Reviews, 23, 895-903 
Nesse, R.M. (2000). Is depression an adaptation? Archives of General Psychiatry, 57, 14-20

Nesse, R.M. (2004). Cliff-edged fitness functions and the persistence of schizophrenia. Behavioral and Brain Sciences, 27, 862-63

Nesse, R.M. (2005). Maladaptation and natural selection. The Quarterly Review of Biology, 80, 62-70

Nettle, D. (2001). Strong Imagination: Madness, Creativity and Human Nature. Oxford, UK: Oxford University Press

Nettle, D. (2004). Evolutionary origins of depression: a review and reformulation. Journal of Affective Disorders, 81, 91-102

Nettle, D. (2006a). The evolution of personality variation in humans and other animals. American Psychologist, 61, 622-31

Nettle, D. (2006b). Reconciling the mutation-selection balance model with the schizotypy-creativity connection. Behavioral and Brain Sciences, 29, 418.

Nettle, D. and Clegg, H. (2006). Schizotypy, creativity and mating success in humans. Proceedings of the Royal Society of London Series B, Biological Sciences, 273, 611-5

Ni, X., Trakalo, J., Valente, J. et al. (2005). Human p53 tumor suppressor gene (TP53) and schizophrenia: case-control and family studies. Neuroscience Letters, 388, 173-8

Nielsen, R., Bustamante, C., Clark, A.G. et al. (2005). A scan for positively selected genes in the genomes of humans and chimpanzees. PLoS Biology, 3, e170

Ørstavik, R.E., Kendler, K.S., Czajkowski, N., Tambs, K. and ReichbornKjennerud, T. (2007). The relationship between depressive personality disorder and major depressive disorder: a population-based twin study. American Journal of Psychiatry, 164, 1866-72

Opgen-Rhein, C., Lencz, T., Burdick, K.E., Neuhaus, A.H., Derosse, P., Goldberg, T.E., and Malhotra, A.K. (2008). Genetic variation in the DAOA gene complex: Impact on susceptibility for schizophrenia and on cognitive performance. Schizophrenia Research, (in press)

Owen, M.J., Craddock, N., Jablensky, A. (2007). The genetic deconstruction of psychosis. Schizophrenia Bulletin, 33, 905-11

Pickard, B.S., Thomson, P.A., Christoforou, A. et al. (2007). The PDE4B gene confers sex-specific protection against schizophrenia. Psychiatric Genetics, 17, 129-33

Potash, J.B. (2006). Carving chaos: genetics and the classification of mood and psychotic syndromes. Harvard Review of Psychiatry, 14, 47-63

Prata, D., Breen, G., Osborne, S., Munro, J., St Clair, D., and Collier, D. (2007). Association of DAO and G72(DAOA)/G30 genes with bipolar 
affective disorder. American Journal of Medical Genetics Part B Neuropsychiatric Genetics, (in press)

Qin, J., Calabrese, P., Tiemann-Boege, I. et al. (2007). The molecular anatomy of spontaneous germline mutations in human testes. PLoS Biology, 5, e224

Rapoport, J.L., Addington, A.M., and Frangou, S. (2005). The neurodevelopmental model of schizophrenia: update 2005. Molecular Psychiatry, 10, 434-49

Reuter, M. and Hennig, J. (2005). Pleiotropic effect of the TPH A779C polymorphism on nicotine dependence and personality. American Journal of Medical Genetics. Part B, Neuropsychiatric Genetics, 134, 20-4

Reuter, M., Roth, S., Holve, K. and Hennig, J. (2006). Identification of first candidate genes for creativity: a pilot study. Brain Research, 1069, 190-7

Rietschel, M., Beckmann, L., Strohmaier, J. et al. (2008). G72 and its association with major depression and neuroticism in large population-based groups from Germany. American Journal of Psychiatry, (in press)

Sabeti, P.C., Schaffner, S.F., Fry, B. et al. (2006). Positive natural selection in the human lineage. Science, 312, 1614-20

Sebat, J., Lakshmi, B., Malhotra, D. et al. (2007). Strong association of de novo copy number mutations with autism. Science, 316, 445-9

Serretti, A., Mandelli, L., Lorenzi, C. et al. (2006). Temperament and character in mood disorders: influence of DRD4, SERTPR, TPH and MAO-A polymorphisms. Neuropsychobiology, 53, 9-16

Sharp, A.J., Locke, D.P., McGrath, S.D. et al. (2005). Segmental duplications and copy-number variation in the human genome. American Journal of Human Genetics, 77, 78-88

Shifman, S., Bhomra, A., Smiley, S. et al. (2008). A whole genome association study of neuroticism using DNA pooling. Molecular Psychiatry, 13, 302-12

Smith, F.M., Garfield, A.S. and Ward, A. (2006). Regulation of growth and metabolism by imprinted genes. Cytogenetic and Genome Research, 113, 279-91

Smits, G. and Kelsey, G. (2006). Imprinting weaves its web. Developmental Cell, 11, 598-9

Smith, D.J., Kyle, S., Forty, L. et al. (2008). Differences in depressive symptom profile between males and females. Journal of Affective Disorders, 108, 279-84

Smith, D.I., Zhu, Y., McAvoy, S. and Kuhn, R. (2006). Common fragile sites, extremely large genes, neural development and cancer. Cancer Letters, 232, 48-57 
Smoller, J.W., Gardner-Schuster, E. and Misiaszek M. (2008). Genetics of anxiety: would the genome recognize the DSM? Depression and Anxiety, $25,368-77$

Song, W., Li, W., Feng, J., Heston, L.L., Scaringe, W.A. and Sommer, S.S. (2008). Identification of high risk DISC1 structural variants with a $2 \%$ attributable risk for schizophrenia. Biochemical and Biophysical Research Communications, 367, 700-6

Soni, S., Whittington, J., Holland, A.J., Webb, T., Maina, E., Boer, H. and Clarke, D. (2007). The course and outcome of psychiatric illness in people with Prader-Willi syndrome: implications for management and treatment. Journal of Intellectual Disability Research, 51, 32-42

Soni, S., Whittington, J., Holland, A.J. et al. (2008). The phenomenology and diagnosis of psychiatric illness in people with Prader-Willi syndrome.

Psychological Medicine, 4, 1-10

Stefanis, N.C., Trikalinos, T.A., Avramopoulos, D. et al. (2007). Impact of schizophrenia candidate genes on schizotypy and cognitive endophenotypes at the population level. Biological Psychiatry, 62, 784-92

Tamminga, C.A. and Holcomb, H.H. (2005). Phenotype of schizophrenia: a review and formulation. Molecular Psychiatry, 10, 27-39

Thomas, P.D. and Kejariwal, A. (2004). Coding single-nucleotide polymorphisms associated with complex vs. Mendelian disease: evolutionary evidence for differences in molecular effects. Proceedings of the National Academy of Sciences, U. S. A. 101, 15398-403

Tycko, B. and Morison, I.M. (2002). Physiological functions of imprinted genes. Journal of Cell Physiology, 192, 245-58

Ubeda, F. and Wilkins, J.F. (2008). Imprinted genes and human disease: an evolutionary perspective. Advances in Experimental Medicinal Biology, 626, 101-15

Voight, B.F., Kudaravalli, S., Wen, X. and Pritchard, J.K. (2006). A map of recent positive selection in the human genome. PLoS Biology, 4, e72

Walsh, T., McClellan, J.M., McCarthy, S.E. et al. (2008). Rare structural variants disrupt multiple genes in neurodevelopmental pathways in schizophrenia. Science, 320, 539-43

Weiss, L.A., Purcell, S., Waggoner, S. et al. (2007). Identification of EFHC2 as a quantitative trait locus for fear recognition in Turner syndrome. Human Molecular Genetics, 16, 107-13

Winantea, J., Hoang, M.N., OhIraun, S. et al. (2006). A summary statistic approach to sequence variation in noncoding regions of six schizophreniaassociated gene loci. European Journal of Human Genetics, 14, 1037-43 
Wolpert, L. (2008). Depression in an evolutionary context. Philosophy, Ethics, and Humanities in Medicine, 3, 8

Yampolsky, L.Y., Kondrashov, F.A. and Kondrashov, A.S. (2005).

Distribution of the strength of selection against amino acid replacements in human proteins. Human Molecular Genetics, 14, 3191-201

Yen, Y.C., Rebok, G.W., Gallo, J.J., Yang, M.J., Lung, F.W. and Shih, C.H. (2007). ApoE4 allele is associated with late-life depression: a populationbased study. American Journal of Geriatric Psychiatry, 15, 858-68

Young, J.H., Chang, Y.P., Kim, J.D. et al. (2005). Differential susceptibility to hypertension is due to selection during the out-of-Africa expansion. PLoS Genetics, 1, e82

Zhao, X., Leotta, A., Kustanovich, V. et al. (2007). A unified genetic theory for sporadic and inherited autism. Proceedings of the National Academy of Sciences USA, 104, 12831-6 